\title{
QT prolongation and cardiac toxicity of new tuberculosis drugs in Europe: a Tuberculosis Network European Trialsgroup (TBnet) study
}

\author{
To the Editor:
}

There is concern that many second-line drugs used to treat multidrug-resistant tuberculosis (MDR-TB) may cause fatal arrhythmias linked to QT interval prolongation. The QT interval, measured on an electrocardiogram (ECG), represents the duration of the ventricular electrical systole. In order to have a more reproducible value across time and heart rates, the QT interval is adjusted according to heart rate (as QTc). Fluoroquinolones and, possibly, clofazimine can prolong the QTc interval $[1,2]$, as do bedaquiline (Bdq) and delamanid (Dlm) [3, 4]. A QTc interval $>500 \mathrm{~ms}$ is considered a risk factor for ventricular arrhythmias such as torsades de pointes (TdP) [5]. Overall, $10-20 \%$ of patients with drug-induced TdP have a genetic predisposition and more than $70 \%$ have at least two other risk factors, like older age, female sex and electrolyte disturbances [6]. In smaller cohorts, no cases of fatal arrhythmia have been reported in association with new tuberculosis (TB) drugs and deaths in Bdq/Dlm registration trials were not linked to prolonged QTc nor arrhythmias [3, 4, 7]. The objective of the study was to evaluate the clinical impact of QTc prolongation and the number of cardiac events in patients receiving Bdq/Dlm treatment for MDR-TB in treatment centres of the Tuberculosis Network European Trialsgroup (TBnet), a clinical research collaboration of the European Respiratory Society, within the World Health Organization (WHO) Europe region.

In December 2016, a cross-sectional online survey was distributed in parallel to members of the TBnet and to a list of TBnet MDR-TB country representatives (chosen because of MDR-TB management expertise and previous participation in TBnet activities) in 45 countries belonging to the WHO Europe region, excluding Central Asia. No more than one participant was accepted from each centre and, in cases of multiple answers from the same centre, participants were contacted to confirm the correct answer. Participants who reported cardiac events in patients receiving MDR-TB treatment containing Bdq/Dlm were contacted to obtain additional information. The survey closed in July 2017. The questionnaire consisted of 10 questions on general information and QTc prolongation management; 32 questions on treatment experience with Bdq/Dlm; and three questions on the use of the Bdq/Dlm combination. The full questionnaire is available online (http://tb-net.org/images/TBnet_completed_projects_QT_survey/TBnet_ Survey_on_QT_interval_monitoring_and_new_TB_drugs.pdf). Ethical approval was provided by the Institutional Review Board of Bligny Hospital, France.

Overall, 61 valid replies from different hospitals in 41 out of 45 (91\%) targeted countries, out of the 53 included in the WHO Europe region, were retained. The most represented countries were Italy (seven participants), Denmark and Spain (four participants each). Most participants worked in teaching/ university hospitals (49\%) and in TB reference hospitals (24\%).

Most physicians (50 out of $61(82 \%)$ ) routinely monitor the QT interval at their centre. Among them, 22 (44\%) perform QT monitoring in all patients undergoing MDR-TB treatment, 20 (40\%) perform it only in patients receiving new drugs or multiple QT-prolonging drugs, and eight (16\%) do not perform it in MDR-TB patients. In MDR-TB patients monitored for QT prolongation, ECGs are repeated weekly

@ERSpublications

Few cardiac arrhythmias and no fatalities were observed in MDR-TB patients treated with bedaquiline and delamanid http://ow.ly/2tC030koXJa

Cite this article as: Guglielmetti L, Tiberi S, Burman M, et al. QT prolongation and cardiac toxicity of new tuberculosis drugs in Europe: a Tuberculosis Network European Trialsgroup (TBnet) study. Eur Respir J 2018; 52: 1800537 [https://doi.org/10.1183/13993003.00537-2018]. 
(23 out of 42 participants (55\%)) or monthly (19 out of 42 participants (45\%)) during treatment, either before administering treatment (34 out of 42 participants (81\%)) or 90-120 min after administration (eight out of 42 participants (19\%)). Overall, 34 participants (68\%) use the QTc interval while $16(32 \%)$ use the uncorrected QT interval. Of the 34 participants measuring QTc, $41 \%$ use the Fridericia correction (QTcF), 23\% use the Bazett correction (QTcB), 18\% use both and 18\% did not know which correction was used. Most physicians would stop Bdq and Dlm in case of QTc interval >500 ms (27 out of 61 participants (44\%)), while 22 out of 61 participants (36\%) would do so only in the case of symptomatic arrhythmias. Furthermore, 12 out of 61 participants (20\%) would also stop the drugs for any prolongation above normal values (470 $\mathrm{ms}$ in women and $450 \mathrm{~ms}$ in $\mathrm{men}$ ).

Overall, 35 participants (57\%) had experience using Bdq and 20 participants (33\%) had experience using Dlm. 15 out of 35 (43\%) of those using Bdq and six out of $20(30 \%)$ of those using Dlm require approval

\section{TABLE 1 List of countries and centres taking part in the study, treatment with new drugs and} QT-related adverse events

\begin{tabular}{|c|c|c|c|c|c|c|}
\hline \multirow[t]{2}{*}{ Country } & \multirow[t]{2}{*}{ Centres } & \multicolumn{3}{|c|}{ Treatment } & \multirow[t]{2}{*}{ QT prolongation" } & \multirow[t]{2}{*}{ Cardiac events ${ }^{+}$} \\
\hline & & Bdq & Dlm & Bdq and Dlm ${ }^{\#}$ & & \\
\hline Albania & 1 & & & & & \\
\hline Armenia & 1 & 110 & 46 & $13(9 / 4)$ & $1(1 / 0)$ & \\
\hline Austria & 1 & 13 & 1 & & & \\
\hline Belarus & 1 & 297 & 33 & $2(2 / 0)$ & & \\
\hline Belgium & 1 & 7 & & & & \\
\hline Bosnia and Herzegovina & 1 & & & & & \\
\hline Bulgaria & 1 & & & & & \\
\hline Croatia & 1 & & & & & \\
\hline Cyprus & 1 & & & & & \\
\hline Czech Republic & 1 & & & & & \\
\hline Denmark & 4 & 9 & 3 & $2(1 / 1)$ & & $1(1 / 0)$ \\
\hline Estonia & 1 & 20 & 13 & & & \\
\hline Finland & 1 & 3 & & & & \\
\hline France & 1 & 70 & 12 & $10(4 / 6)$ & $5(5 / 0)$ & \\
\hline Georgia & 1 & 292 & 45 & & & $1(0 / 1)$ \\
\hline Germany & 3 & 39 & 15 & $4(1 / 3)$ & & \\
\hline Greece & 1 & & & $1(1 / 0)$ & & \\
\hline Hungary & 1 & & & & & \\
\hline Iceland & 1 & & & & & \\
\hline Ireland & 1 & 2 & 1 & $1(1 / 0)$ & & \\
\hline Italy & 7 & 14 & & & & \\
\hline Kosovo & 1 & & & & & \\
\hline Latvia & 1 & 53 & 34 & $4(0 / 4)$ & & \\
\hline Lithuania & 1 & 11 & 9 & & & \\
\hline Luxembourg & 1 & 1 & & & & \\
\hline Macedonia & 1 & & & & & \\
\hline Moldova & 1 & 30 & & & $1(1 / 0)$ & \\
\hline Montenegro & 1 & & & & & \\
\hline Norway & 1 & & & & & \\
\hline Poland & 1 & & & & & \\
\hline Portugal & 1 & 1 & 1 & & & \\
\hline Romania & 2 & 40 & & & & \\
\hline Russia & 2 & 13 & & & $1(1 / 0)$ & \\
\hline Serbia & 1 & & & & & \\
\hline Slovakia & 1 & & & & & \\
\hline Spain & 4 & & & & & \\
\hline Sweden & 1 & 4 & 1 & & & \\
\hline Switzerland & 1 & 1 & 1 & & $1(0 / 1)$ & \\
\hline The Netherlands & 3 & 10 & 4 & $1(1 / 0)$ & & \\
\hline UK & 3 & 3 & 1 & & & \\
\hline Ukraine & 1 & 1 & & & & \\
\hline Total & 61 & 1044 & 220 & $38(20 / 18)$ & $9(8 / 1)$ & $2(1 / 1)$ \\
\hline
\end{tabular}

Bdq: bedaquiline; DIm: delamanid. " : values in parentheses are for concomitant/sequential treatment; $\overbrace{\text { : }}$ patients who had to stop a new drug for QT prolongation (Bdq/Dlm); ${ }^{+}$: patients with severe cardiac events while receiving a new drug (Bdq/Dlm). 
from another health body to use the new drug, mainly national consiliums. While most physicians used new drugs for no longer than 24 weeks, nine out of 35 (26\%) and four out of 20 (20\%) had prescribed treatment durations up to 20 months for Bdq and Dlm, respectively.

Physicians who used Bdq had previously treated a limited number of patients with this drug (median: four patients (interquartile range (IQR) 2-25)). Out of a total of 1044 Bdq-treated patients, this drug had to be stopped in eight cases following QT prolongation (0.77\% (95\% CI 0.04-1.57)). One cardiac event was reported $(0.10 \%(95 \%$ CI $0.01-0.63))$ in a 55-year old diabetic patient from Somalia, treated for lymph node MDR-TB, who developed an asymptomatic first-degree atrioventricular block associated with QT interval prolongation $(\mathrm{QTcF}=460 \mathrm{~ms})$ while receiving moxifloxacin, clofazimine, cycloserine, Bdq, ethambutol and prothionamide. The QT prolongation was recorded in association with an overdose of Bdq during the continuation phase of treatment, where the patient took $600 \mathrm{mg}$ of Bdq daily for 5 days, instead of $200 \mathrm{mg}$ three times a week, due to a misunderstanding of the prescription. The patient was hospitalised and, after 3 weeks, his QTcF normalised and he continued Bdq until the end of treatment.

Overall, 220 patients were treated with Dlm, with each physician seeing a median of two patients (IQR 113). Dlm was stopped in one case following QT prolongation (0.45\% (95\% CI 0.02-2.89)) and one significant cardiac event was reported $(0.45 \%$ (95\% CI $0.02-2.89)$ ) in a 23 -year old female patient from Georgia. The patient, treated for pulmonary MDR-TB with Dlm, linezolid, clofazimine, capreomycin, cycloserine and pyrazinamide, and receiving metoprolol, experienced multiple episodes of hypokalaemia and concomitant QT prolongation (QTcF $>500 \mathrm{~ms}$ ) with transient palpitations and dyspnoea but no evidence of ECG alterations, which resolved after temporary treatment interruption.

Overall, the use of the new drugs in association with other QT-prolonging drugs was common (26 out of $35(75 \%)$ with Bdq and 18 out of 20 (90\%) with Dlm), with 10 out of $35(29 \%)$ and 9 out of $20(45 \%)$ physicians reporting the use of Bdq and Dlm with two or more QT-prolonging drugs. In particular, 14 out of 61 physicians (24\%) used the Bdq-Dlm combination in concomitant treatment and 11 out of 61 (19\%) used it sequentially, for a total of 38 patients exposed to both drugs.

QT monitoring during MDR-TB treatment is commonly performed in Europe, although the frequency of ECG testing and the management of QT prolongation vary substantially across centres and countries. Experience with new drugs still appears to be limited, especially for Dlm. Although most participants reported the use of the new drugs in association with other QT-prolonging agents, Bdq and Dlm were stopped in only a few cases because of QT prolongation. Few clinically relevant cardiac adverse events and no fatal cases were reported. These findings support published results [8-10] and recent data from a Phase III clinical trial testing Dlm versus a placebo [11]. In addition, a relevant proportion of physicians used the new drugs for treatment for more than 6 months $[12,13]$ and in combination with QT-interval prolonging agents $[14,15]$. Limitations of this study include retrospective data collection, the analysis of aggregate data, incomplete coverage of the WHO Europe region with over-representation of Western Europe, bias linked to surveys which may lead to underestimating the number of events and the absence of sudden death in the questionnaire.

In conclusion, QTc interval monitoring is regularly performed by physicians treating patients with Bdq and/or Dlm in the WHO Europe region. The frequency of clinically relevant cardiac events associated with the use of these drugs is low. Among 1044 patients treated with Bdq and 220 patients treated with Dlm as part of a MDR-TB treatment regimen, not a single case of a fatal cardiac event was observed.

Lorenzo Guglielmetti $\circledast^{1,2,3}$, Simon Tiberi ${ }^{4,5}$, Matthew Burman ${ }^{5}$, Heinke Kunst ${ }^{5}$, Christian Wejse $\oplus^{6}$, Tamar Togonidze ${ }^{7}$, Graham Bothamley ${ }^{8}$ and Christoph Lange ${ }^{9,10,11}$ for the TBnet ${ }^{12}$

${ }^{1}$ APHP, Centre National de Référence des Mycobactéries et de la Résistance des Mycobactéries aux Antituberculeux (CNR-MyRMA), Bactériologie-Hygiène, Hôpitaux Universitaires Pitié Salpêtrière-Charles Foix, Paris, France. ${ }^{2}$ Sorbonne Université, Université Pierre et Marie Curie 06, Unité 1135, Team E13 (Bactériologie), CR7 INSERM, Centre d'Immunologie et des Maladies Infectieuses, Paris, France. ${ }^{3}$ Sanatorium, Centre Hospitalier de Bligny, Briis-sous-Forges, France. ${ }^{4}$ Division of Infection, Royal London Hospital, Barts Health NHS Trust, London, UK. ${ }^{5}$ Blizard Institute, Barts and The London School of Medicine and Dentistry, Queen Mary University of London, London, UK. ${ }^{6}$ Dept of Infectious Diseases, Aarhus University Hospital, Aarhus, Denmark. ${ }^{7}$ National Center for Tuberculosis and Lung Diseases, Tbilisi, Georgia. ${ }^{8}$ Dept of Respiratory Medicine, Homerton University Hospital, London, UK. ${ }^{9}$ Division of Clinical Infectious Diseases, Medical Clinic Research Center Borstel, German Center for Infection Research (DZIF), Clinical Tuberculosis Center, Borstel, Germany. ${ }^{10}$ International Health/Infectious Diseases, University of Lübeck, Lübeck, Germany. ${ }^{11}$ Dept of Medicine, Karolinska Institute, Stockholm, Sweden. ${ }^{12}$ Additional members of the TBNET QTc survey collaboration can be found in the acknowledgements section.

Correspondence: Lorenzo Guglielmetti, Laboratoire de Bactériologie-Hygiène, Faculté de Médecine Pierre et Marie Curie (UPMC Paris 6), 91 Boulevard de l'hôpital, 75634 Paris Cedex 13, France. E-mail: lorenzo.guglielmetti@gmail.com

Received: March 172018 | Accepted after revision: May 262018 
Additional members of the TBNET QTc survey collaboration: Hasan Hafizi (Albania), Ohanna Kirakosyan (Armenia), Rudolf Rumetshofer (Austria), Alena Skrahina (Belarus), Inge Muylle (Belgium), Hasan Zutic (Bosnia and Herzegovina), Roumiana Markova (Bulgaria), Mateja Jankovic (Croatia), Zsuzsanna Gyorfy (Czech Republic), Georgiades Georgios (Cyprus), Åse Bengård Andersen, Pernille Ravn and Shailesh Kolekar (Denmark), Manfred Danilovits (Estonia), Tuula Vasankari (Finland), Mathilde Fréchet-Jachym (France), Nana Kiria and Zaza Avaliani (Georgia), Nils Wetzstein and Korkut Avsar (Germany), George Dimopoulos (Greece), Ildiko Horvath (Hungary), Már Kristjánsson (Iceland), Anne Marie McLaughlin (Ireland), Elena Chiappini, Marco Confalonieri, Paola Del Bravo, Gina Gualano, Ilaria Motta, Emanuele Pontali and Simone Tunesi (Italy), Xhevat Kurhasani (Kosovo), Liga Kuksa (Latvia), Laimute Jacunskiene (Lithuania), Wirtz Gil (Luxembourg), Zorica Nanovikj (Macedonia), Dumitru Chesov (Moldova), Olivera Bojovic (Montenegro), Wiel De Lange, Cecile Magis-Escurra and Jakko Van Ingen (Netherlands), Mogens Jensenius (Norway), Katarzyna Kruczak (Poland), Raquel Duarte (Portugal), Traian Mihaescu and Elmira Ibraim (Romania), Anna Kaluzhenina and Grigory Volchenkov (Russian Federation), Dragica Pesut (Serbia), Ivan Solovic (Slovakia), José-María García-García, Rafael Luque-Márquez, Adrián Sánchez-Montalvá and M. Luiza de Souza Galvao (Spain), Maria Norrby (Sweden), Otto Schoch (Switzerland), Andrii Dudnyk (Ukraine), Moerida Belton and Martin Dedicoat (UK).

Author contributions: L. Guglielmetti made a substantial contribution to the conception and design of the survey, to the analysis and interpretation of data for the work, wrote the manuscript, critically revised the manuscript for important intellectual content and gave final approval for the current version to be published. S. Tiberi, M. Burman, G. Bothamley and C. Lange made a substantial contribution to the conception and design of the survey, to the interpretation of data for the work, critically revised the manuscript for important intellectual content and gave final approval for the current version to be published. All other authors critically revised the manuscript for important intellectual content and gave final approval for the current version to be published. All authors agreed to be accountable for all aspects of the work in ensuring that questions related to the accuracy or integrity of any part of the work are appropriately investigated and resolved.

Conflict of interest: C. Lange reports personal fees for independent lectures at sponsored symposia from Chiesi, Gilead, Abbvie, MSD, Becton Dickinson, Janssen, Lucane, Novartis and Thermofisher, outside the submitted work.

\section{References}

1 Haverkamp W, Kruesmann F, Fritsch A, et al. Update on the cardiac safety of moxifloxacin. Curr Drug Saf 2012; 7: 149-163.

2 Choudhri SH, Harris L, Butany JW, et al. Clofazimine induced cardiotoxicity-a case report. Lepr Rev 1995; 66: 63-66.

3 Diacon AH, Pym A, Grobusch MP, et al. Multidrug-resistant tuberculosis and culture conversion with bedaquiline. N Engl J Med 2014; 371: 723-732.

4 Gupta R, Geiter LJ, Hafkin J, et al. Delamanid and QT prolongation in the treatment of multidrug-resistant tuberculosis. Int J Tuberc Lung Dis 2015; 19: 1261-1262.

5 Drew BJ, Ackerman MJ, Funk M, et al. Prevention of torsade de pointes in hospital settings: a scientific statement from the American Heart Association and the American College of Cardiology Foundation. Circulation 2010; 121: $1047-1060$

6 Trinkley KE, Page RL II, Lien H, et al. QT interval prolongation and the risk of torsades de pointes: essentials for clinicians. Curr Med Res Opin 2013; 29: 1719-1726.

7 Gler MT, Skripconoka V, Sanchez-Garavito E, et al. Delamanid for multidrug-resistant pulmonary tuberculosis. N Engl J Med 2012; 366: 2151-2160.

8 Yoon HY, Jo KW, Nam GB, et al. Clinical significance of QT-prolonging drug use in patients with MDR-TB or NTM disease. Int J Tuberc Lung Dis 2017; 21: 996-1001.

9 Pontali E, Sotgiu G, Tiberi S, et al. Cardiac safety of bedaquiline: a systematic and critical analysis of the evidence. Eur Respir J 2017; 50: 1701462.

10 Borisov SE, Dheda K, Enwerem M, et al. Effectiveness and safety of bedaquiline-containing regimens in the treatment of MDR- and XDR-TB: a multicentre study. Eur Respir J 2017; 49: 1700387.

11 Geiter LJ. Delamanid global clinical database and Phase 3 trial results. The 48th Union World Conference on Lung Health (Satellite Session: October 13, 2017), Guadalajara, Mexico, October 11-14, 2017.

12 Guglielmetti L, Jaspard M, Le Dû D, et al. Long-term outcome and safety of prolonged bedaquiline treatment for smultidrug-resistant tuberculosis. Eur Respir J 2017; 49: 1601799.

13 Kuksa L, Barkane L, Hittel N, et al. Final treatment outcomes of multidrug- and extensively drug-resistant tuberculosis patients in Latvia receiving delamanid-containing regimens. Eur Respir J 2017; 50: 1701105.

14 Guglielmetti L, Barkane L, Le Dû D, et al. Safety and efficacy of exposure to bedaquiline-delamanid in multidrug-resistant tuberculosis: a case series from France and Latvia. Eur Respir J 2018; 51: 1702550.

15 Maryandyshev A, et al. Bedaquiline and delamanid combination treatment of 5 patients with pulmonary extensively drug-resistant tuberculosis. Emerg Infect Dis 2017; 23: 1718-1721. 